\title{
User Experience of a Social Media Based Knowledge Sharing System in Industry Work
}

\author{
Susanna Aromaa ${ }^{1(凶)}$, Maria Tsourma ${ }^{2}$, Stylianos Zikos ${ }^{2}$, \\ Eija Kaasinen ${ }^{1}$, Mariia Kreposna ${ }^{3}$, Anastasios Drosou ${ }^{2}$, \\ and Dimitrios Tzovaras ${ }^{2}$ \\ 1 VTT Technical Research Centre of Finland Ltd., Tampere, Finland \\ \{susanna.aromaa, eija.kaasinen\}@vtt.fi \\ 2 Information Technologies Institute Centre for Research and Technology \\ Hellas, Thessaloniki, Greece \\ \{mtsourma, szikos, drosou, dimitrios.tzovaras\}@iti.gr \\ ${ }^{3}$ FINN-POWER Oy, Seinäjoki, Finland \\ mariia.kreposna@primapower.com
}

\begin{abstract}
With increasing automation, the work in manufacturing industry is getting knowledge intensive: workers need to solve complex problems to keep systems running. The knowledge needed in these situations often cannot be found in ready-made manuals but it is tacit knowledge possessed by co-workers. This paper presents a user study of a knowledge sharing platform. The platform integrates production information and social media features, thus connecting discussions to specific machine states or error situations. The platform was evaluated with factory workers in a workshop. The results show that integration of production information and social media features such as messaging is valuable, as it proactively provides practical solutions to the problem at hand. The results also show that the use of social media features in industrial environment requires changes in organizational policies, processes and culture. The findings of this study can be considered when developing social media applications for industrial use.
\end{abstract}

Keywords: Knowledge sharing - Social media $\cdot$ User evaluation - Production • Gamification · Industry work

\section{Introduction}

Industry 4.0 factories require smart and skilled operators because work in manufacturing industry is getting knowledge intensive. Recovering from an alarm situation requires knowledge but access to the knowledge can be challenging as situations are different and most of them are not documented in the official manuals for end-users. The operators recover from alarms by learning by doing or by contacting their colleagues. In these cases, the shared knowledge remains known by few. Therefore, there is a need to share the knowledge more widely to make good practices visible and accessible for all. 
A promising approach to support knowledge sharing is social media technologies. Social media can be defined as a combination of various forms of media content that are publicly available in a platform and that are created by end-users in a participatory and collaborative fashion [1]. Currently, the use of social media technologies and social messenger systems in the work context has grown as organizations have begun to realize that social media might aid organizational knowledge sharing [2, 3]. Aromaa et al. [4] report user studies of an augmented reality (AR) based, knowledge-sharing system in industrial maintenance. In their study, the social media features for adding and sharing one's own comments, notes and pictures embedded with AR on physical objects was seen very useful.

The use of social media to support knowledge sharing in work environments has still challenges to overcome. Mere installation of social media platforms is not enough: success in adoption and effectiveness in full exploitation will be dependent on reconfiguration and redesign of the whole socio-technical and managerial system [5]. According to Kassner et al. [6], "no manufacturing-specific approach for integrating social media into the shop floor workspace currently exists".

Key challenge in knowledge sharing is how to motivate the employees to participate. Swacha [7] has proposed gamification as an effective way to increase motivation to share knowledge. Gamification is commonly defined as the use of gaming elements to improve user experience and user engagement in non-game services and applications [8]. It is widely used in the domains of marketing, health and education. However, in factory floor environments, it is a new concept [9].

This paper describes the design and user study of a knowledge sharing platform targeted to factory floor workers. The platform integrates social media features to the production information. This facilitates connecting discussions e.g. to certain machine alarms, as the relevant discussions can be shown to the user when an alarm occurs. Messages can be complemented with photos and videos to visualize proposed solutions. In addition, gamification features aim to motivate workers to share knowledge.

\section{Social Media Platform}

The Social Media Platform (SoMeP) is a web-based application, which aims to increase interaction, communication and socialization among workers through the use of various social media technologies such as discussion forums, instant messaging, news feeds, and gamification. The main novelty of SoMeP is that it integrates social media features with production information. By using SoMeP, workers are able to share multimedia content, exchange opinions with their colleagues, as well as find and provide solutions to problems. SoMeP is able to receive alarms from machines of the production line and visualize them in real-time, as shown in Fig. 1. When a user faces an alarm situation, s/he can find information about the alarm codes on the production view available in SoMeP. By selecting a specific alarm in the view, the system makes a query to the knowledge base for relevant discussions in the forum based on the alarm ID and machine ID, and presents the list of relevant discussions found. The list can be filtered by creation time and popularity. The user is able to add a new post related to the 
specific alarm ID and to attach a file in a discussion. In addition, user feedback mechanisms such as text replies to posts and 'Like' buttons are implemented in SoMeP.

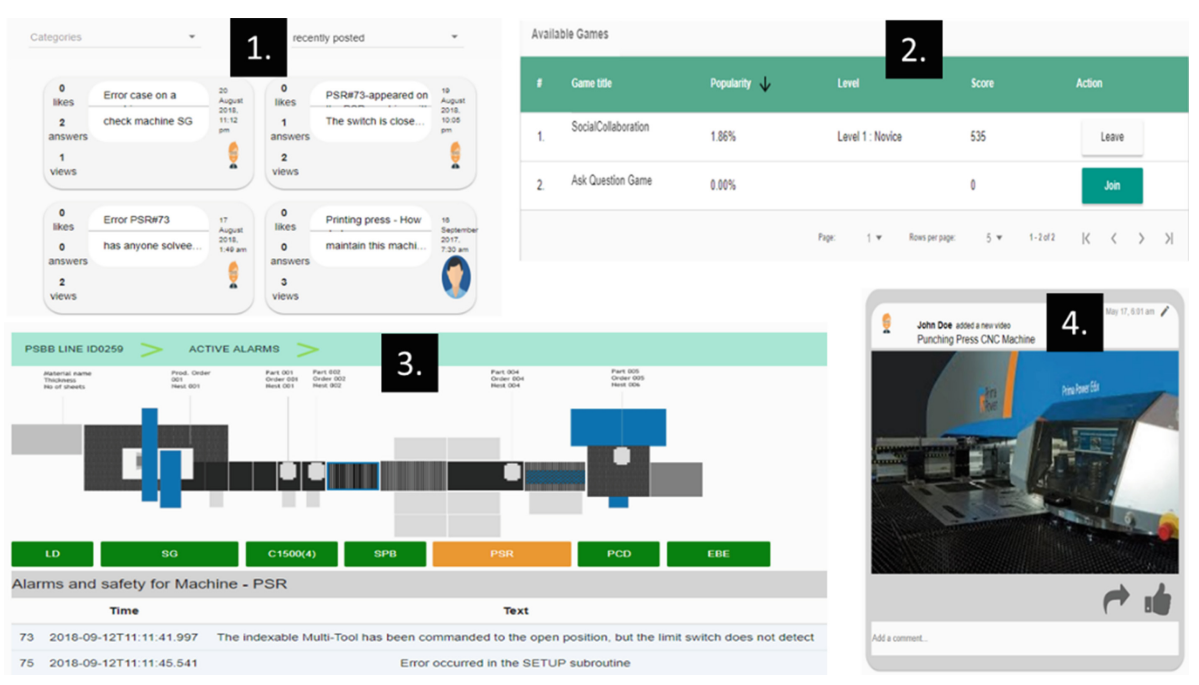

Fig. 1. SoMeP screenshots: (1) discussions in the forum, (2) user participation in games, (3) production status monitoring and machine error alarms and (4) news feed.

A gamification module is integrated into the SoMeP, aiming at increasing workers' motivation and participation in knowledge sharing. It implements a rating system, through which users can earn points and awards when sharing their knowledge and when others assess the shared knowledge valuable. Moreover, predefined levels can be unlocked based on the collected points, indicating user engagement. Awards in the form of badges can be earned when a user has performed certain number of actions. Actions, rules and awards are defined in games that are created by the administrator, targeting to motivate users to participate in discussions and share good solutions to work-related problems. A personal profile dashboard informs the user about the collected points per game, current level and earned awards (Fig. 1).

SoMeP is intended to be deployed inside the local network of the factory and can be accessed via PC as well as mobile devices such as tablets located at the shop floor. The platform supports two types of users, administrators and regular users. All users are able to login to the system, view current active alarms, search for already stored solutions, provide feedback, and upload new information on how a specific alarm can be resolved. In alarm situations, the system displays the alarm code and the "official" guidance related to it. If the user cannot resolve the alarm with the guidance, s/he can open the SoMeP discussion related to the alarm. If the discussion does not help, either s/he can request support via instant messaging or e-mail. Administrators can additionally perform content moderation operations and set up games. 


\section{Methods}

Nine male participants took part to the evaluation. Their age range was 24-56 years (average 46 years). They were all working in a company that manufactures highly automated metal sheet processing machines. Their professions were automation designer, technical specialist, electrician, technician, two engineers, quality expert, manager and head of design. Four of them were active messenger users and five did have some experience of messenger systems (e.g., WhatsApp, Facebook messenger).

A focus group approach was used as a data collection method. The SoMeP usability, pros/cons and development ideas were discussed in two groups. In addition, a project specific evaluation framework [10] was used when creating questionnaires and discussion topics. The questionnaires were used to collect participant demographics, UX (with smiley faces on 5 point Likert scale), the system usability scale (SUS) [11] and knowledge sharing questions (5 point Likert scale). Discussions were recorded via a voice recorder. Data was analyzed qualitatively.

First, the participants signed the consent forms and filled in demographic information. The SoMeP was introduced briefly to the whole group with a live demo. Then, the participants were divided into two focus groups with 5 and 4 participants, respectively. Three facilitators were collecting data: one in the first group and two in the second group. Within both groups, participants were encouraged to try out different features of the SoMeP running on a PC such as discussion forums, instant messaging, news feed, production alarm views, and the games. After trying each feature, participants gave comments and facilitators asked questions related to the pros and cons as well as further development ideas. Each participant filled in an evaluation questionnaire at the end of the focus group.

\section{Results}

\subsection{User Experience, Usability and User Acceptance}

The overall user experience of the SoMeP was good: most of the participants felt using the system as a positive experience. In addition, the usability of the system was rated to be on a good level: the SUS score was 75. It can be perceived that the participants would like to use the system frequently. They agreed that the system was easy to use and they would not need guidance from an expert. They thought that the system was not complex, nor did it have too many inconsistencies. Most of them agreed that the system would be easy to learn to use and felt quite confident while using it.

Regarding user acceptance, the participants agreed that integrating discussions with production system information was beneficial. In addition, they agreed that the system could increase knowledge sharing within the factory. Most of the participants thought that seeing how many had liked the comments was good when evaluating the usefulness of the comments. However, 3 participants neither agreed nor disagreed with it. The ability to add videos to the discussions was referred as beneficial.

The participants gave contradictory comments regarding the gamification features. They mentioned that gamification could motivate workers to share knowledge but on 
the other hand, one participant said that gathering points does not belong to the work. In the knowledge sharing platform, the gamification scores are seen only by the worker him/herself. Still the participants wondered if other people could see their personal scores. The participants were concerned that managers may think that workers are only playing and not doing their work. They also thought that there might be cultural differences on accepting games, and gamification might not work in less developed countries due to workers' skill level and workplace hierarchy.

\subsection{Comments and Development Ideas}

The participants suggested some usability improvements during the discussions. For example, more text could be visible of the messages before opening them, the text and icons could be larger throughout the platform, and several alarms should be shown simultaneously.

The participants claimed that the production view was the best feature in the system. They commented that it is useful because it was possible to search messages based on the alarm code. However, they noticed that for a successful search, the users should write alarm codes in similar formats. The participants thought that the filtering and sorting of messages could be improved to manage all the messages better. Adding videos to the messages was seen useful: with them novice workers can concretely see how to perform an assembly task. However, the participants wondered how the overall content moderation would be organized.

In addition, the SoMeP could be useful in knowledge sharing between different groups (e.g., worker-worker, worker-maintenance, worker-management, companycustomer). The participants pointed that the knowledge sharing community would probably influence the knowledge sharing activity and the content. This is because sharing within a familiar, limited group, is easier.

The cultural differences were discussed too. In some less developed countries, it is hard to imagine workers sharing knowledge via the system. In addition, cultural differences exist between companies for example in the form of different policies for knowledge sharing. The participants also raised a concern that some people do not want to share their expertise to others, e.g. because they may fear losing their power as acknowledged experts.

The participants commented that SoMeP could be useful if a company has lot of rented workforce. They suggested that by using this platform new people could ask questions easily even if they do not know who to ask. In addition, it would help company's workers learn to know new workers if their profiles (name and photo) are accessible through the platform.

\section{Conclusions}

It can be concluded that most of the participants liked SoMeP and found it useful and easy to use. Particularly, integration of production information and messages was seen valuable. The participants thought that SoMeP would increase knowledge sharing but they were questioning whether gamification is the right way to motivate workers to 
participate. The use of social media features in industrial environments requires changes in organizational policies, processes and cultures. It is important to define the community who shares the knowledge and who else can access the shared knowledge. Content moderation is needed to make sure that false information is not shared. As a future work, we plan to exploit the feedback received from the workers in order to improve the usability of the platform, re-design the interface and enrich it with additional features. In addition, the acceptance of gamification use in a production environment needs to be evaluated thoroughly. In general, social media technologies should be studied in a long-term use to understand better the needs for content supervision and company policies.

Acknowledgments. This project has received funding from the European Union's Horizon 2020 research and innovation programme under grant agreement No. 723277 (project Factory2Fit). This paper reflects only the authors' view and the Commission is not responsible for any use that may be made of the information it contains. The authors are grateful to all researchers, concepts' developers and company representatives who have contributed to and supported the work presented in this publication.

\section{References}

1. Kaplan, A.M., Haenlein, M.: Users of the world, unite! The challenges and opportunities of social media. Bus. Horiz. 53(1), 59-68 (2010)

2. Leonardi, P.M.: Ambient awareness and knowledge acquisition: using social media to learn 'who knows what' and 'who knows whom'. MIS Q. 39(4), 747-762 (2015)

3. Jarrahi, M.H., Sawyer, S.: Social technologies, informal knowledge practices, and the enterprise. J. Org. Comp. Elect. Com. 23(1-2), 110-137 (2013)

4. Aromaa, S., Väätänen, A., Hakkarainen, M., Kaasinen, E.: User experience and user acceptance of an augmented reality based knowledge-sharing solution in industrial maintenance work. In: 8th International Conference on Applied Human Factors and Ergonomics, pp. 145-156. Springer, Cham (2018)

5. Denyer, D., Parry, E., Flowers, P.: "Social", “open" and "participative"? Exploring personal experiences and organisational effects of enterprise 2.0 use. Long Range Plann. 44(5-6), 375-396 (2011)

6. Kassner, L., Hirmer, P., Wieland, M., Steimle, F., Königsberger, J., Mitschang, B.: The social factory: connecting people, machines and data in manufacturing for context-aware exception escalation. In: 50th Hawaii International Conference on System Sciences, pp. 1673-1682 (2017)

7. Swacha, J.: Gamification in knowledge management: motivating for knowledge sharing. Polish J. Manag. Stud. 12(2), 150-160 (2015)

8. Deterding, S., Sicart, M., Nacke, L., O’Hara, K., Dixon, D.: Gamification: using game design elements in non-gaming contexts. In: CHI'11 Extended Abstracts on Human Factors in Computing Systems, pp. 2425-2428. ACM (2011)

9. Korn, O., Funk, M., Schmidt, A.: Design approaches for the gamification of production environments: a study focusing on acceptance. In: 8th ACM International Conference on Pervasive Technologies Related to Assistive Environments, p. 6. ACM (2015) 
10. Kaasinen, E., Liinasuo, M., Schmalfuß, F., Koskinen, H., Aromaa, S., Heikkilä, P., Honka, A., Mach, S., Malm, T.: A worker-centric design and evaluation framework for operator 4.0 solutions that support work well-being. In: Human Work Interaction Design. Designing Engaging Automation. HWID 2018. IFIP Advances in Information and Communication Technology, 544, pp. 263-282. Springer, Cham (2018)

11. Brooke, J.: SUS-A quick and dirty usability scale. In: Usability Evaluation in Industry, pp. 189-194. Taylor \& Francis, London (1996)

Open Access This chapter is licensed under the terms of the Creative Commons Attribution 4.0 International License (http://creativecommons.org/licenses/by/4.0/), which permits use, sharing, adaptation, distribution and reproduction in any medium or format, as long as you give appropriate credit to the original author(s) and the source, provide a link to the Creative Commons license and indicate if changes were made.

The images or other third party material in this chapter are included in the chapter's Creative Commons license, unless indicated otherwise in a credit line to the material. If material is not included in the chapter's Creative Commons license and your intended use is not permitted by statutory regulation or exceeds the permitted use, you will need to obtain permission directly from the copyright holder.

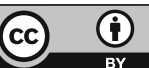

